\title{
Mechanisms Contributing to Adverse Cardiovascular Events in Patients with Type 2 Diabetes Mellitus and Stage 4 Chronic Kidney Disease Treated with Bardoxolone Methyl
}

\author{
Melanie P. Chin ${ }^{a}$ Scott A. Reisman ${ }^{a}$ George L. Bakris ${ }^{b} \quad$ Megan O'Grady ${ }^{a}$ \\ Peter G. Lindec Peter A. McCullough ${ }^{\text {d, e }}$ David Packham $^{\text {h }}$ Nosratola D. Vaziri $^{f}$ \\ Keith W. Ward ${ }^{a}$ David G. Warnock ${ }^{g}$ Colin J. Meyer ${ }^{a}$ \\ ${ }^{a}$ Reata Pharmaceuticals, Irving, Tex., b The University of Chicago Medicine, Chicago, Ill., ' AbbVie Pharmaceuticals, \\ North Chicago, III., ${ }^{d}$ Baylor University Medical Center, Baylor Heart and Vascular Institute, Baylor Jack and Jane \\ Hamilton Heart and Vascular Hospital, Dallas, Tex., ${ }^{\mathrm{e}}$ The Heart Hospital, Plano, Tex., ${ }^{\mathrm{f}}$ University of California, \\ Irvine, Calif., and ${ }^{9}$ Department of Medicine, University of Alabama at Birmingham, Birmingham, Ala., USA; \\ ${ }^{\mathrm{h}}$ Melbourne Renal Research Group and Department of Nephrology, Royal Melbourne Hospital and Medicine, \\ University of Melbourne, Melbourne, Vic., Australia
}

\section{Key Words}

Bardoxolone methyl · Type 2 diabetes mellitus · Chronic kidney disease $\cdot$ Randomized controlled trial $\cdot$ Serious adverse event $\cdot$ Heart failure $\cdot$ Endothelin · Fluid overload B-type natriuretic peptide

\begin{abstract}
Background: Bardoxolone methyl, an Nrf2-activating and nuclear factor-кB-inhibiting semisynthetic oleanane triterpenoid compound, was evaluated in a phase 3 trial (BEACON) in patients with type 2 diabetes mellitus (T2DM) and stage 4 chronic kidney disease (CKD). The trial was terminated because of an increase in heart failure events in the bardoxolone methyl group, many of which appeared related to fluid retention. Thus, additional analyses were conducted to explain these serious adverse events. Methods: Patients $(\mathrm{n}=$ $2,185)$ were randomized to receive once-daily bardoxolone methyl (20 mg) or placebo. Twenty-four-hour urine collec-
\end{abstract}

\begin{tabular}{ll}
\hline KARGER & $\begin{array}{l}\text { ( } 2014 \text { S. Karger AG, Basel } \\
0250-8095 / 14 / 0396-0499 \$ 39.50 / 0 \quad \text { Karger }\end{array}$ \\
E-Mail karger@karger.com & $\begin{array}{l}\text { This is an Open Access article licensed under the terms of the } \\
\text { www.karger.com/ajn }\end{array}$ \\
& $\begin{array}{l}\text { Creative Commons Attribution-NonCommercial 3.0 Un- } \\
\text { ported license (CC BY-NC) (www.karger.com/OA-license), } \\
\text { applicable to the online version of the article only. Distribu- } \\
\text { tion permitted for non-commercial purposes only. }\end{array}$
\end{tabular}

tions were analyzed in a subset of the BEACON population and from a separate, open-label pharmacology study in patients with stage $3 \mathrm{~b} / 4 \mathrm{CKD}$ and T2DM administered $20 \mathrm{mg}$ bardoxolone methyl once daily for 56 consecutive days. Results: Bardoxolone-methyl-treated patients in the BEACON substudy had a clinically meaningful reduction in urine volume and sodium excretion at week 4 relative to baseline $(p<0.05)$, and a separate study revealed that decreased sodium excretion and urine output occurred in some patients with stage 4 CKD but not those with stage 3b CKD. The clinical phenotype of fluid overload and heart failure in BEACON was similar to that observed with endothelin receptor antagonists in advanced CKD patients, and preclinical data demonstrate that bardoxolone methyl modifies endothelin signaling. Conclusions: The totality of the evidence suggests that through modulation of the endothelin pathway, bardoxolone methyl may pharmacologically promote acute sodium and volume retention and increase blood pressure in patients with more advanced CKD.

(c) 2014 S. Karger AG, Base 


\section{Introduction}

Bardoxolone methyl and its analogs are semi-synthetic oleanane triterpenoid compounds, which activate the transcription factor nuclear factor-erythroid-2-related factor 2 (Nrf2) and induce a potent cytoprotective and anti-inflammatory phenotype $[1,2]$. Through interaction with the Nrf2 repressor molecule, Kelch-like ECH-associated protein 1 (Keap1), bardoxolone methyl facilitates translocation of Nrf2 to the nucleus, where it binds to antioxidant response elements in the promoter region of its target genes, leading to induction of many antioxidant and cytoprotective enzymes and related proteins $[3,4]$. In fact, Nrf2 transcriptionally controls approximately $90 \%$ of all antioxidant genes, making it an important innate defense mechanism against oxidative stress [5]. Bardoxolone methyl is also a potent inhibitor of the nuclear factor$\kappa \mathrm{B}(\mathrm{NF}-\kappa \mathrm{B})$ inflammatory pathway through both direct (i.e. inhibition of IKK $\beta$ kinase activity) $[6,7]$ and indirect mechanisms (i.e. detoxification of reactive oxygen species). Because of this dual mechanism of action, bardoxolone methyl is hypothesized to have potential therapeutic relevance in a variety of disease settings involving oxidative stress and inflammation.

Bardoxolone methyl and close analogs demonstrate efficacy in rodent models of kidney disease. Twelve weeks of daily administration of the bardoxolone methyl analog CDDO-dhTFEA (or RTA dh404) significantly decreased mean arterial pressure and reduced glomerulosclerosis, interstitial fibrosis, and inflammation in rats with chronic kidney disease (CKD) induced by 5/6 nephrectomy [8]. Both bardoxolone methyl and its analog CDDO-Im have demonstrated protective effects against cisplatin-induced acute nephrotoxicity $[9,10]$. Analogs of bardoxolone methyl also ameliorate angiotensin II-induced kidney injury and estimated glomerular filtration rate (eGFR) declines in rats [11], as well as protect against iron nitrilotriacetate-induced kidney injury [12].

Several phase 2 clinical trials have also revealed that bardoxolone methyl improves several measures of kidney function. A phase 2, double-blind, randomized, placebocontrolled trial enrolling patients with type 2 diabetes mellitus (T2DM) and mild to moderate CKD demonstrated a significant increase in eGFR with these effects sustained for 52 weeks [13]. On the basis of these data, a multinational, randomized, double-blind, placebo-controlled phase 3 outcomes trial in patients with T2DM and stage $4 \mathrm{CKD}$ (Bardoxolone Methyl Evaluation in Patients with Chronic Kidney Disease and Type 2 Diabetes: The Occurrence of Renal Events, BEACON), was designed and initiated [14].
The BEACON trial was terminated for safety concerns in response to recommendations from the independent data monitoring committee [14]. The primary reason for the recommendation was a significantly increased risk of heart failure requiring hospitalization, which also increased the composite cardiovascular outcome (nonfatal myocardial infarction, nonfatal stroke, hospitalization for heart failure, or death from cardiovascular causes) in the bardoxolone methyl arm compared to the placebo arm. No increased risk of heart failure with bardoxolone methyl treatment was observed in prior phase 1 and phase 2 clinical studies $[13,15]$ or in preclinical testing in cynomolgus monkeys [16]. However, in BEACON, elevated B-type natriuretic peptide (BNP), which was measured blindly, and prior hospitalization for heart failure were associated with increased risk for heart failure in stage 4 CKD patients treated with bardoxolone methyl [17].

Suppression of endothelin signaling in patients with advanced CKD with other pharmacological agents results in a pattern of heart failure similar to that observed with bardoxolone methyl treatment in BEACON. A comprehensive investigation, including additional analyses from BEACON and other clinical studies with bardoxolone methyl, as well as preclinical experiments, was conducted to explore potential bardoxolone methyl-mediated effects on fluid handling and modulation of the endothelin pathway as potential mechanism(s) for the increased risk of heart failure in BEACON patients.

\section{Methods}

\section{Clinical Studies}

Phase 3 BEACON Study

Previous publications describe the BEACON trial design, patient demographics, and baseline characteristics [14, 18, 19]. Briefly, a total of 2,185 patients with T2DM and stage 4 CKD were randomized 1:1 to once-daily administration of bardoxolone methyl $(20 \mathrm{mg})$ or placebo. Median time on study was 9 months. The primary efficacy outcome of the study was the time-to-first event in the composite outcome defined as end-stage renal disease (ESRD; need for chronic dialysis, renal transplantation, or renal death) or cardiovascular death. Secondary efficacy outcomes included the change in eGFR, time-to-first hospitalization for heart failure or death due to heart failure, and time-to-first event of the composite consisting of nonfatal myocardial infarction, nonfatal stroke, hospitalization for heart failure, or cardiovascular death. An independent Events Adjudication Committee (EAC), blinded to study treatment assignment, evaluated whether ESRD events, cardiovascular events, strokes, and fatalities met pre-specified definitions of primary and secondary end points, as described in the EAC charter [14]. A subset of the BEACON population $(\mathrm{n}=174,8.0 \%)$ consented to additional 24-hour assessments of ambulatory blood pressure monitoring (ABPM) and 24-hour urine collections at se- 
lected visits. The study protocol was approved by Institutional Review Boards at participating study sites and was registered at ClinicalTrials.gov (NCT01351675).

Phase 2 Study

In a separate, open-label pharmacology study, 24 patients with T2DM and stage 3b/4 CKD (eGFR 15-45 ml/min/1.73 $\mathrm{m}^{2}$ ) were administered $20 \mathrm{mg}$ bardoxolone methyl once daily for 56 consecutive days. 24-hour urine collections were completed on study days 1 and 56 and analyzed for urinary electrolytes. The study protocol was approved by Institutional Review Boards at participating study sites and was registered at ClinicalTrials.gov (NCT01549769).

\section{Preclinical Studies}

In vitro Study

Rat proximal tubular cells (NRK-52E cells) were treated with vehicle (dimethyl sulfoxide, $0.1 \%$ final concentration) or $10-300$ $\mathrm{nM}$ of bardoxolone methyl in the absence or presence of $10 \mathrm{mg} / \mathrm{ml}$ bovine serum albumin. After $24 \mathrm{~h}$, cell culture media was collected and assessed for concentrations of secreted endothelin-1 (ET-1) using a commercially available ELISA kit (R\&D Systems, Inc., Minneapolis, Minn., USA).

\section{5/6 Nephrectomy Model of CKD}

As has been previously described [16], bardoxolone methyl is subject to rodent-specific adverse metabolism rendering rodents unsuitable for direct evaluation of pharmacologic effects of this compound; therefore, RTA dh404 was used as a close structural analog for these rodent studies. Male Sprague-Dawley rats were subjected to a sham operation (sham controls, $n=6$ ), or $5 / 6$ nephrectomy to induce CKD [8]. Briefly, RTA dh404 (2 mg/kg) or vehicle (sesame oil) was administered orally once daily for 12 weeks to rats with CKD ( $\mathrm{n}=9$ per group). After 12 weeks, the kidney or remnant kidney was immediately removed, frozen in liquid nitrogen, and stored at $-80^{\circ} \mathrm{C}$ until analysis. Expression of endothelin receptors type $\mathrm{A}$ and $\mathrm{B}\left(\mathrm{ET}_{\mathrm{A}}\right.$ and $\left.\mathrm{ET}_{\mathrm{B}}\right)$ in the kidney were assessed by Western blot using antibodies against $\mathrm{ET}_{\mathrm{A}}$ and $\mathrm{ET}_{\mathrm{B}}$ (Alomone Labs, Jerusalem, Israel). Cytosolic fractions were prepared and analyzed as previously described [20]. An antibody against GAPDH (Santa Cruz Biotechnology Inc., Dallas, Tex., USA) was used for measurements of the housekeeping protein. All experiments were approved by the University of California-Irvine Institutional Committee for the Use and Care of Experimental Animals.

Nonhuman Primate Study

Healthy female cynomolgus monkeys, aged 2-5 years, were treated with vehicle (sesame oil) or bardoxolone methyl $30 \mathrm{mg} / \mathrm{kg} /$ day for 28 days [16]. A separate group of animals was treated with bardoxolone methyl for 28 days and then allowed to recover for 14 days. Following the treatment period, animals were euthanized $24 \mathrm{~h}$ after the final dose of bardoxolone methyl, and kidneys were collected, rinsed in ice-cold PBS, and frozen in liquid nitrogen. $\mathrm{ET}_{\mathrm{A}}$ and $\mathrm{ET}_{\mathrm{B}}$ protein expression, using antibodies from Genetex Inc. (Irvine, Calif., USA) were assessed using immunohistochemistry with methods similar to those previously described [16]. $\mathrm{ET}_{\mathrm{A}}$ and $\mathrm{ET}_{\mathrm{B}}$ staining intensity of $\times 2.5$ magnification photomicrographs was quantified utilizing ImageJ software v1.46 with the Densitometry 1 plug-in, both available from the National Institute of Health (http://rsbweb.nih.gov/ij/index.html). Animal welfare

Bardoxolone Methyl Adverse Events Mechanisms for this study was compliant with the U.S. Department of Agriculture's Animal Welfare Act (9 CFR Parts 1,2, and 3), and the study protocol was reviewed and approved by an Institutional Animal Care and Use Committee.

\section{Statistical Analysis}

Preclinical data were analyzed by one-way analysis of variance (ANOVA) followed by Duncan's multiple range post hoc test using Sigmaplot 12.0 (Systat Inc., San Jose, Calif., USA).

\section{Results}

\section{Clinical Presentation of Heart Failure Events in BEACON}

An increase in fatal and nonfatal heart failure events was the major finding that led to the early termination of BEACON: $96 / 1,088$ (8.8\%) patients randomized to bardoxolone methyl patients versus 55/1,097 (5.0\%) patients randomized to placebo, corresponding to a hazard ratio of 1.83 (95\% CI 1.32-2.55) [14]. Over $80 \%$ of the excess heart failure events observed in the bardoxolone methyl group occurred within the first 3-4 weeks after initiation of treatment. Review of the narrative descriptions for these events provided in hospital admission notes indicates that heart failure in bardoxolone methyl-treated patients was often preceded by rapid weight gain (increase of several kilograms immediately after randomization to the active treatment arm). In addition, many hospital records revealed reports of dyspnea, peripheral edema, central/pulmonary edema on imaging, elevated blood pressure and heart rate, unchanged serum creatinine values, and preserved left ventricular ejection fraction. The clinical presentation of these events adjudicated as heart failure in the BEACON trial suggested that there was early-onset fluid retention in the setting of preserved ejection fraction and elevated blood pressure.

\section{Assessment of Clinical Characteristics Associated with}

\section{Postrandomization Increases in BNP}

As previously reported, post hoc analyses using an increase in BNP (as a surrogate for fluid retention or increased left ventricular wall stress) showed that bardoxolone methyl-treated patients experienced a significantly larger increase in BNP than placebo-treated patients [14]. The change in BNP (baseline to week 24) was unrelated to baseline eGFR, changes in eGFR, or changes in albu$\mathrm{min} /$ creatinine ratio (ACR; table 1 ). However, in bardoxolone methyl-treated patients only, baseline ACR was significantly correlated with week 24 changes from baseline in BNP $(\mathrm{p}<0.01$; table 1$)$. This suggests that the propen- 
Table 1. Correlations between changes from baseline in BNP at week 24 and other clinical parameters in bardoxolone methyl vs. placebo patients in BEACON

\begin{tabular}{lllr}
\hline Parameter & Treatment & $\begin{array}{l}\text { Correlation } \\
\text { coefficient }\end{array}$ & $\begin{array}{l}\mathrm{p} \\
\text { value }^{\mathrm{a}}\end{array}$ \\
\hline Week 24 BNP change & & & \\
Baseline log ACR & placebo & 0.05 & 0.47 \\
& BARD & 0.20 & $<0.01$ \\
WK24 log ACR change & placebo & 0.14 & 0.04 \\
& BARD & $<0.01$ & 0.96 \\
Baseline eGFR & placebo & -0.03 & 0.69 \\
Week 24 eGFR change & BARD & -0.10 & 0.16 \\
& placebo & 0.04 & 0.57 \\
& BARD & -0.03 & 0.70 \\
\hline
\end{tabular}

Includes only patients with baseline and week $24 \mathrm{BNP}, \mathrm{ACR}$, and eGFR data [placebo, $\mathrm{n}=216$; bardoxolone methyl (BARD), $\mathrm{n}=211]$.

${ }^{\text {a }}$ Fisher's z-transformation of the correlations was used to compare correlations between placebo and bardoxolone methyl groups.

sity for fluid retention may be associated with baseline severity of kidney disease, as defined by albuminuria status, and was not primarily associated with the general changes in renal function, as assessed by eGFR.

\section{Twenty-Four-Hour Urine Collections}

Twenty-four-hour urine collections from the ABPM substudy revealed clinically meaningful reductions in urine volume and excretion of sodium at week 4 relative to baseline in the bardoxolone methyl-treated patients $(-247 \pm 71 \mathrm{ml}, \mathrm{p}<0.05$; table 2$)$ that were not observed in placebo-treated patients.

Additionally, in a separate study in patients with type 2 diabetes and stage $3 \mathrm{~b} / 4 \mathrm{CKD}, 8$ weeks of treatment with $20 \mathrm{mg}$ of bardoxolone methyl (NCT01549769) led to significant increases in urinary sodium retention as well as water volume retention in patients with stage $4 \mathrm{CKD}$ compared to those with stage $3 \mathrm{~b}$ CKD (table 2). Patients with stage $4 \mathrm{CKD}$ experienced reductions in both parameters, whereas patients with stage $3 \mathrm{~b}$ CKD did not appear to retain sodium.

\section{Effects of Bardoxolone Methyl on Blood Pressure}

Blood Pressure Changes in CKD Patients

As previously described, mean changes from baseline in systolic and diastolic blood pressures, based on the average of triplicate standardized blood pressure cuff mea- surements, were acutely increased in the bardoxolone methyl group relative to the placebo group in BEACON [14]. Mean \pm SD increases of $1.9 \pm 14.0 \mathrm{~mm} \mathrm{Hg}$ in systolic and $1.4 \pm 7.4 \mathrm{~mm} \mathrm{Hg}$ in diastolic blood pressures were noted in the bardoxolone methyl group by week 4 (the first postrandomization assessment). ABPM measurements at week 4 showed more pronounced blood pressure increases in bardoxolone methyl-treated patients relative to placebo-treated patients [14].

In a separate, open-label, dose-ranging study in 131 patients with type 2 diabetes and stage $3 \mathrm{~b} / 4 \mathrm{CKD}$ (NCT01053936), no dose-related trend in blood pressure changes or change at any individual dose level was noted following 85 consecutive days of treatment at doses ranging from 2.5 to $30 \mathrm{mg}$ daily of bardoxolone methyl. Prespecified analysis of blood pressure data stratified by CKD stage suggests that bardoxolone methyl-treated patients with stage $4 \mathrm{CKD}$ (approx. one-third of the patients) tended to have mean increases in blood pressure relative to baseline levels, whereas bardoxolone methyltreated patients with stage $3 \mathrm{~b}$ CKD had no apparent change (table 3 ). This finding directly parallels the differential effects bardoxolone methyl treatment has on sodium and volume retention in stage $3 \mathrm{~b}$ versus stage $4 \mathrm{CKD}$ patients (table 2), suggesting a link between volume retention and blood pressure increases in this particular patient population receiving bardoxolone methyl. Although sample sizes in the dose groups stratified by CKD stage are small, the results show a similar relationship whether median or mean values are analyzed, and suggest that the effect of bardoxolone methyl treatment on blood pressure may be related to CKD stage.

Blood pressure values from a phase $2 \mathrm{~b}$ study with bardoxolone methyl (BEAM, NCT00811889), which used an earlier formulation of the drug and employed a titration design, were highly variable and despite noted increases in some bardoxolone methyl treatment groups, no clear dose-related trend was observed in blood pressure [13].

\section{Blood Pressure in Healthy Volunteers}

To determine if bardoxolone methyl has a primary effect on blood pressure, intensive blood pressure monitoring was employed in a separate 'Thorough QT Study', which was conducted in 179 healthy volunteers (NCT01689116). In both bardoxolone methyl-treated groups, one given the therapeutic dose, $20 \mathrm{mg}$, and the other given the supratherapeutic dose of $80 \mathrm{mg}$, the change in blood pressure did not differ from changes in placebo-treated patients after 6 days of once-daily administration and trended down at later time points (fig. 1). 
Table 2. Changes from baseline in urinary parameters in bardoxolone methyl patients

\begin{tabular}{|c|c|c|c|c|}
\hline & \multicolumn{2}{|c|}{ BEACON ABPM substudy } & \multicolumn{2}{|c|}{ Patient pharmacokinetic study } \\
\hline & $\mathrm{PBO}$ (stage $4 \mathrm{CKD}$ ) & BARD (stage 4 CKD) & BARD (stage 4 CKD) & BARD (stage 3b CKD) \\
\hline & WK4 $\Delta$ & WK4 $\Delta$ & WK8 $\Delta$ & WK8 $\Delta$ \\
\hline \multicolumn{5}{|l|}{ Urine volume } \\
\hline Patients & 71 & 63 & 6 & 9 \\
\hline Mean \pm SE, $\mathrm{ml} / 24 \mathrm{~h}$ & $-110 \pm 71$ & $-247 \pm 71^{*}$ & $-597 \pm 140^{*}$ & $174 \pm 251^{\dagger}$ \\
\hline \multicolumn{5}{|l|}{ Urinary sodium } \\
\hline Patients & 62 & 57 & 6 & 9 \\
\hline Mean \pm SE, $\mathrm{mmol} / 24 \mathrm{~h}$ & $-11 \pm 9$ & $-27 \pm 9^{*}$ & $-79 \pm 20 *$ & $-5 \pm 17^{\dagger}$ \\
\hline
\end{tabular}

Urine volume and urinary sodium from the BEACON 24-hour ABPM substudy and an open-label pharmacology (patient pharmacokinetic) study in patients with T2D and stage 3b/4 CKD (NCT01549769). In the patient pharmacokinetic study, patients were treated with $20 \mathrm{mg}$ bardoxolone methyl once daily for 56 consecutive days; posttreatment follow-up visit occurred on study day 84 . Analyses include only patients with baseline and week 4 or week 8 data. Data were analyzed by Student's two-tailed t test. PBO = Placebo.

${ }^{*} \mathrm{p}<0.05$ for week 4 or week 8 vs. baseline values within each sub group. ${ }^{\dagger} \mathrm{p}<0.05$ for week 8 changes in stage $3 \mathrm{~b}$ vs. stage $4 \mathrm{CKD}$ patients in the patient pharmacokinetic study.

\section{Comparison of Bardoxolone Methyl Clinical}

\section{Phenotype versus That of Endothelin Receptor}

\section{Antagonists}

The data presented in prior sections suggest that bardoxolone methyl promotes fluid retention in patients who are at greatest risk of developing heart failure. Therefore, renal mechanisms that affect volume status, including effects on the renin angiotensin-aldosterone system (RAAS) and perturbation of transtubular ion gradients, were explored to determine if any of these matched the clinical phenotype observed with bardoxolone methyl. For example, the effects on potassium balance seen with RAAS activation [21] were not observed with bardoxolone methyl. This is further exemplified by the unchanged levels of urine or serum potassium in the BEACON ABPM substudy. Therefore, the totality of the evidence suggests that endothelin antagonism may be responsible for the hemodynamic effects seen with bardoxolone methyl in BEACON.

The most directly analogous clinical data the authors were able to identify for comparison of the effects of known endothelin pathway modulators with the BEACON study are those with the endothelin receptor antagonist avosentan. Avosentan was studied in stage 3-4 CKD patients with diabetic nephropathy in the ASCEND study, a large outcomes study to assess the time to first doubling of serum creatinine, ESRD, or death [22]. As in BEACON, the ASCEND study was terminated early because of an imbalance in heart failure hospitalization and fluid overload events. Using definitions for fluid over-
Table 3. Changes from baseline in systolic and diastolic blood pressure in patients with T2DM and stage $3 \mathrm{~b} / 4$ CKD stratified by baseline CKD stage dosed with bardoxolone methyl

\begin{tabular}{clrcc}
\hline Dose & $\begin{array}{l}\text { CKD } \\
\text { stage }\end{array}$ & Patients & \multicolumn{2}{l}{$\begin{array}{l}\text { Mean day 85 BP change } \pm \text { SD, } \\
\mathrm{mm} \mathrm{Hg}\end{array}$} \\
\cline { 3 - 5 } & & & SBP & DBP \\
\hline \multirow{2}{*}{$2.5 \mathrm{mg}$} & $3 \mathrm{~b}$ & 10 & $0.0 \pm 13.9$ & $1.0 \pm 6.5$ \\
& 4 & 4 & $0.3 \pm 21.9$ & $-1.5 \pm 7.7$ \\
$5 \mathrm{mg}$ & $3 \mathrm{~b}$ & 19 & $-2.1 \pm 8.6$ & $-1.3 \pm 6.2$ \\
& 4 & 5 & $0.5 \pm 20.3$ & $-1.4 \pm 12.4$ \\
$10 \mathrm{mg}$ & $3 \mathrm{~b}$ & 20 & $-4.2 \pm 15.4$ & $-0.3 \pm 5.9$ \\
& 4 & 4 & $6.1 \pm 13.4$ & $3.6 \pm 9.0$ \\
$15 \mathrm{mg}$ & $3 \mathrm{~b}$ & 26 & $-2.2 \pm 16.9$ & $-1.3 \pm 7.8$ \\
& 4 & 22 & $5.0 \pm 13.1$ & $-0.6 \pm 9.1$ \\
$30 \mathrm{mg}$ & $3 \mathrm{~b}$ & 3 & $-0.4 \pm 23.9$ & $-1.8 \pm 6.7$ \\
& 4 & 9 & $9.7 \pm 21.8$ & $4.7 \pm 7.5$ \\
\hline
\end{tabular}

Pre-planned analysis of systolic blood pressure (SBP) and diastolic blood pressure (DBP) from an open-label, dose-ranging study in T2D patients with stage 3b/4 CKD (NCT01053936) was performed. Patients were administered $2.5,5,10,15$, or $30 \mathrm{mg}$ doses of bardoxolone methyl once daily for 85 days.

load-related (serious and nonserious adverse) events specified in ASCEND, a post hoc Kaplan-Meier analysis for fluid overload-related events in BEACON revealed striking similarities in the timing of fluid overload (fig. 2). In both studies, avosentan- or bardoxolone methyl-induced fluid overload events manifested soon after treatment initiation, and event curves appeared to maintain 

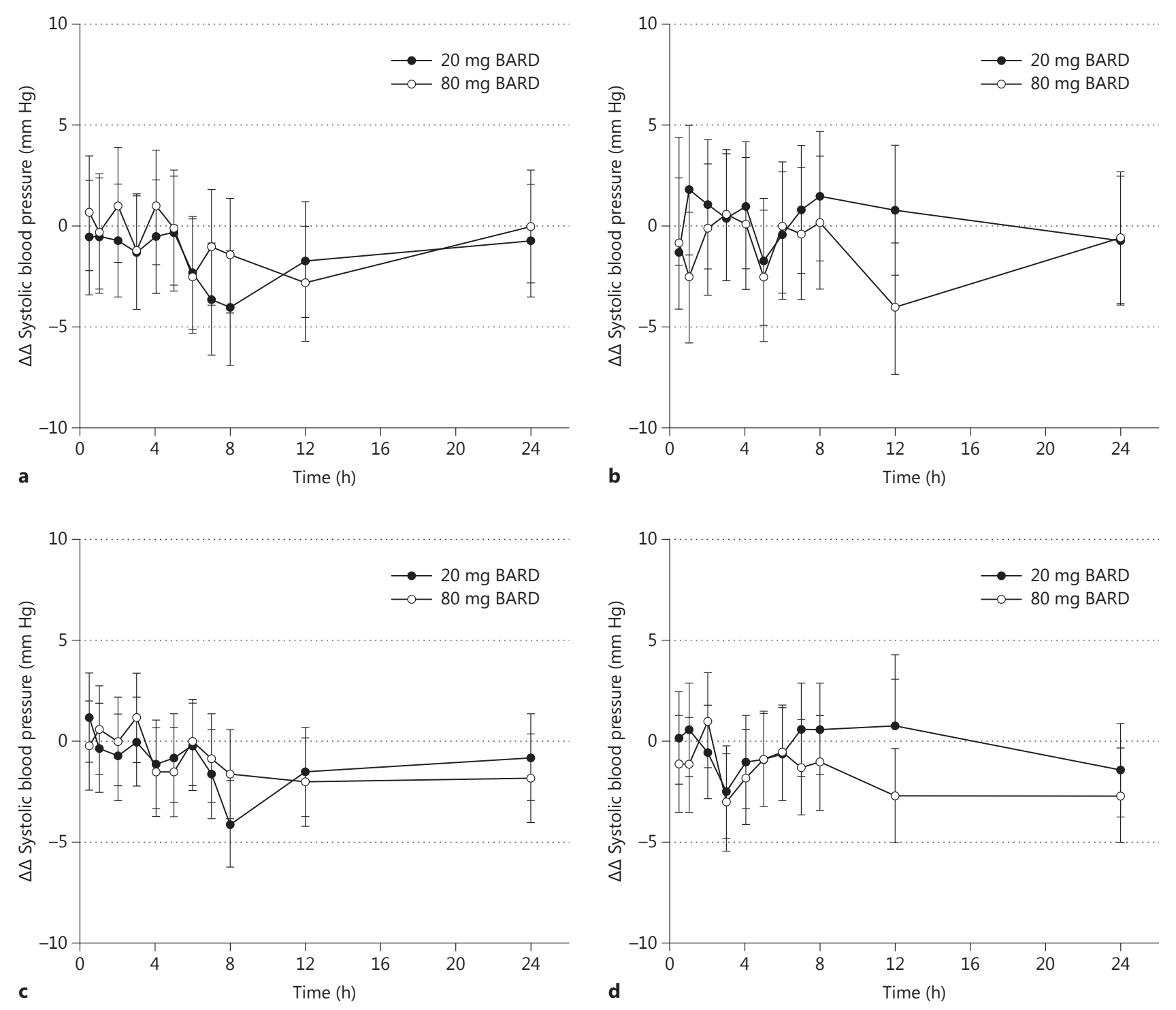

Fig. 1. Placebo-corrected changes from baseline in systolic and diastolic blood pressure in healthy volunteers administered bardoxolone methyl. Results from a multiple-dose, randomized, doubleblind, placebo-controlled thorough QT study in healthy volunteers (NCT01689116). Subjects were treated with placebo, 20 or 80

mg of bardoxolone methyl (BARD), or $400 \mathrm{mg}$ of moxifloxacin (active comparator) once daily for 6 consecutive days. Data are mean placebo-corrected systolic and diastolic blood pressure changes from baseline ( $\pm 90 \% \mathrm{CI}) 0-24 \mathrm{~h}$ after dose on study day 1 (a, c) and study day $6(\mathbf{b}, \mathbf{d})$.

trajectories parallel to the respective placebo arms after the first month of treatment [22].

\section{Bardoxolone Methyl Modulates Endothelin Signaling}

Because the endothelin pathway is activated in the kidney by albumin and other noxious stimuli, the effect of bardoxolone methyl on secretion of ET-1 from proximal

tubular epithelial cells was assessed. Treatment with bardoxolone methyl resulted in a significant and dose-dependent decrease in basal secretion of ET-1 (fig. 3a). Moreover, bardoxolone methyl also significantly and dose-dependently decreased albumin-induced ET-1 secretion, even to undetectable levels at the highest concentration tested $(300 \mathrm{nM})$. 


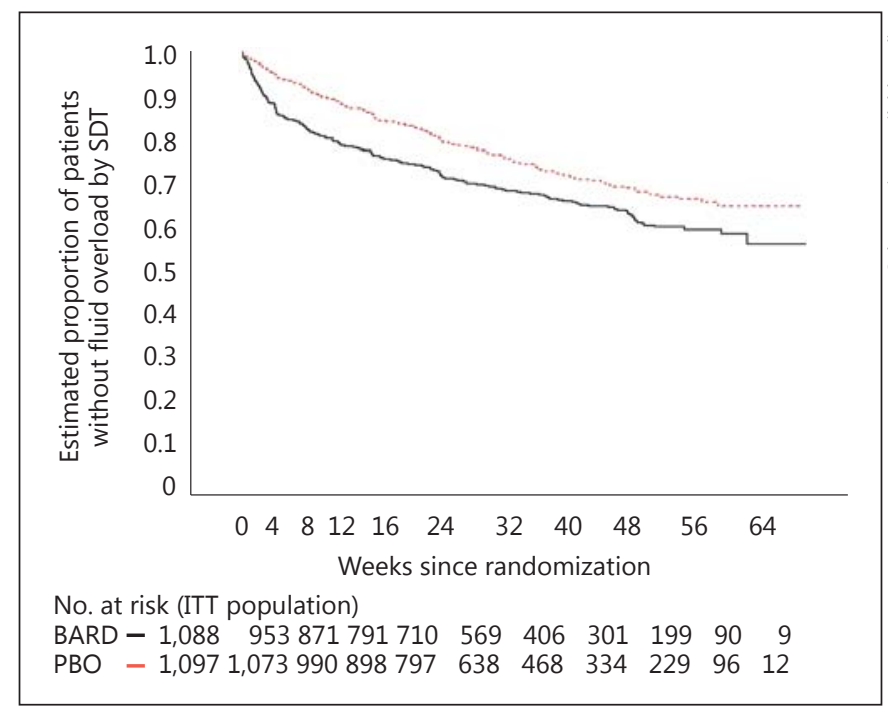

Fig. 2. Kaplan-Meier plots for fluid overload events in BEACON (intent-to-treat, ITT, population). Kaplan-Meier survival analysis for fluid overload events in BEACON. Using definitions specified in ASCEND, fluid overload events were taken from the adverse event reports of the local investigators. Individual signs and symptoms on the adverse event forms indicating fluid overload included: heart failure, edema, fluid overload, fluid retention, hypervolemia, dyspnea, pleural and pericardial effusions, ascites, weight increase, pulmonary rales, and pulmonary edema. $\mathrm{PBO}=$ Placebo; SDT $=$ study drug termination (October 12, 2012).

In a 5/6 nephrectomy model of CKD in rats, 12 weeks of daily administration of the bardoxolone methyl analog (RTA dh404), after surgery, suppressed expression of proinflammatory and profibrotic mediators [8] and promoted a vasodilatory endothelin receptor phenotype, whereby the induction of the $\mathrm{ET}_{\mathrm{A}}$ receptor was completely suppressed, and expression of the $\mathrm{ET}_{\mathrm{B}}$ receptor was partially restored to levels observed in control animals (fig. 3b).

Expression of $\mathrm{ET}_{\mathrm{A}}$ and $\mathrm{ET}_{\mathrm{B}}$ was also assessed in a pharmacology study of bardoxolone methyl in healthy monkeys (fig. 3c). After 28 days of treatment, bardoxolone methyl markedly and significantly reduced protein expression of $\mathrm{ET}_{\mathrm{A}}$ specifically in the renal papilla and medulla by approximately $65 \%$ relative to vehicle-treated controls. In a separate group of animals treated for 28 days and then allowed to recover for an additional 14 days, the protein expression of $\mathrm{ET}_{\mathrm{A}}$ returned to vehicle levels. This study also showed bardoxolone methyl does not affect $\mathrm{ET}_{\mathrm{B}}$ expression in healthy primates (data not shown). Notably, bardoxolone methyl did not increase NT-pro-BNP levels relative to vehicle controls in plasma samples collected from monkeys on day 28 of treatment (fig. 3d). Thus, in the kidney of healthy monkeys, where bardoxolone methyl has been shown to induce protein and mRNA expression of Nrf2 target genes [16], the drug also pharmacologically modulates the endothelin pathway but does not increase BNP. Importantly, the site of $\mathrm{ET}_{\mathrm{A}}$ regulation occurred in the renal medulla, where the endothelin pathway is known to affect sodium and water homeostasis in the renal tubules. Collectively these data suggest that the potent activation of Nrf2-related genes with bardoxolone methyl is associated with suppression of endothelin signaling.

\section{Discussion}

An increased number of heart failure events that appear to be fluid overload in etiology were observed in bardoxolone methyl-treated patients in BEACON. The pattern and clinical characteristics of patients that were hospitalized for these events in the BEACON trial suggested an acute, physiologic effect that precipitated fluid overload relatively early in the course of bardoxolone methyl treatment. Elevated baseline BNP and prior hospitalization for heart failure were associated with hospitalization or death due to heart failure in BEACON [17]. Additionally, as a surrogate of fluid retention or increased left ventricular wall strain, BNP increases were significantly greater in bardoxolone methyl-treated patients than in placebo-treated patients at week 24 [14]. The BNP increases correlated directly with baseline ACR, suggesting that the propensity for fluid retention may be associated with baseline albuminuria status, presumably as a reflection of the baseline severity of the kidney disease in these patients.

Urinary sodium excretion data from the BEACON ABPM substudy patients revealed a clinically meaningful reduction in urine volume and excretion of sodium at week 4 relative to baseline in the bardoxolone methyltreated patients only; in another study, urinary sodium levels and water excretion were significantly reduced only in stage 4 CKD patients, but not in stage $3 \mathrm{~b}$ CKD patients. Together, these data suggest that bardoxolone methyl may affect sodium and water volume handling, with retention of both more pronounced in patients with stage 4 CKD compared with patients with earlier stage CKD.

In a related finding, blood pressure was also increased in the bardoxolone methyl group relative to the placebo group in BEACON [14]. Prespecified blood pressure analysis in healthy volunteer studies demonstrated no 


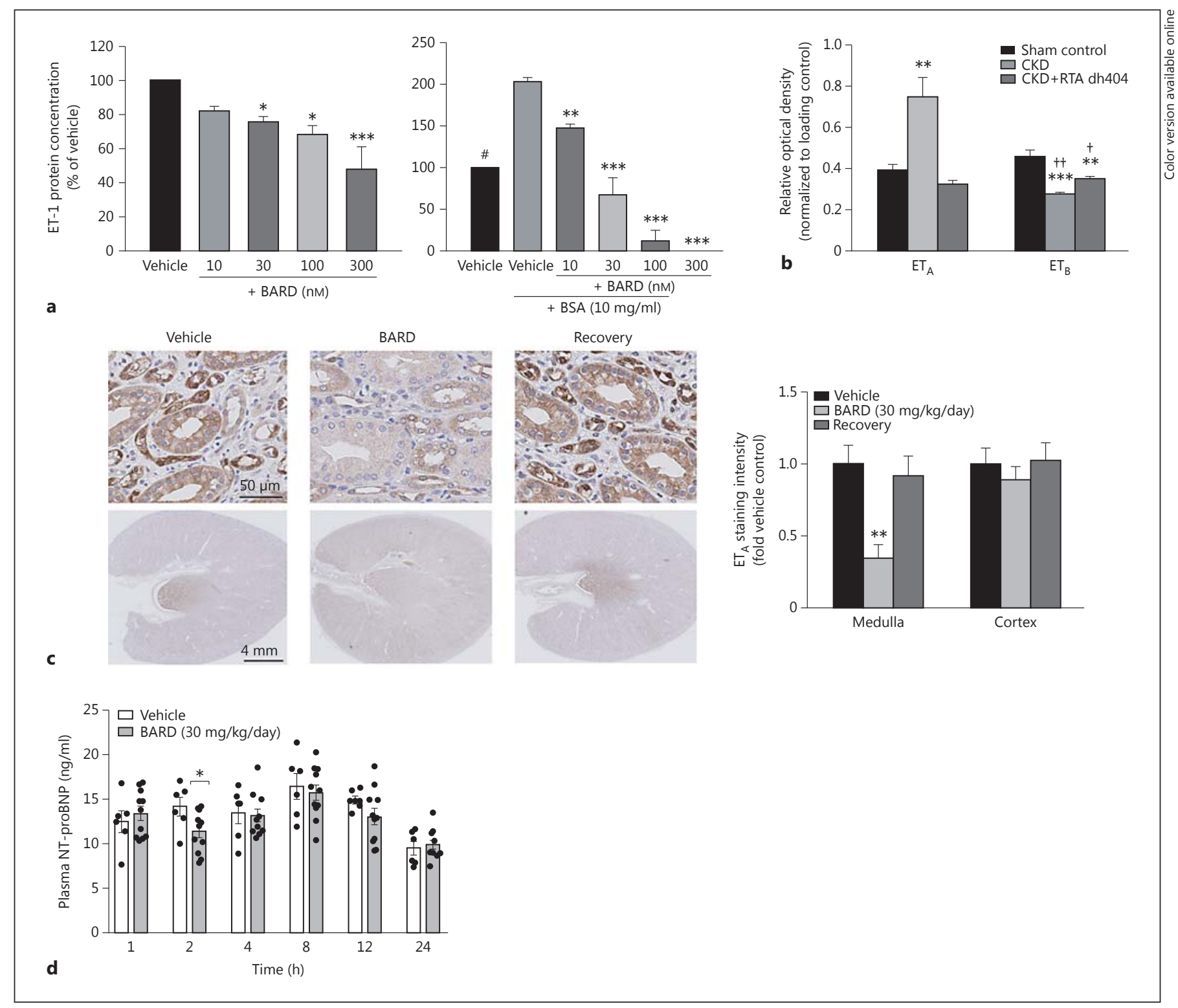

Fig. 3. Effect of bardoxolone methyl on endothelin expression and NT-proBNP levels. a Basal (left) and inducible (right) endothelin-1 (ET-1) protein concentrations (as \% vehicle control) secreted into media from rat proximal tubule cells treated with vehicle or bardoxolone methyl (10-300 nM) in the absence or presence of 10 $\mathrm{mg} / \mathrm{ml}$ bovine serum albumin (BSA). ${ }^{*} \mathrm{p}<0.05,{ }^{* *} \mathrm{p}<0.01$, ${ }^{* * *} \mathrm{p}<0.001$ vs. vehicle control. ${ }^{\#} \mathrm{p}<0.001$, unstimulated group is significantly different from stimulated vehicle group. b Protein expression of $\mathrm{ET}_{\mathrm{A}}$ and $\mathrm{ET}_{\mathrm{B}}$ receptors in the kidney of Sprague-Dawley rats that were subjected to a sham operation (sham control, $n=6$ ) or $5 / 6$ nephrectomy to induce CKD. CKD rats were treated with RTA dh404 (2 mg/kg) or vehicle once daily for 12 weeks ( $\mathrm{n}=$ 9/group). ${ }^{* *} \mathrm{p}<0.01,{ }^{* * *} \mathrm{p}<0.001$ vs. control; ${ }^{\dagger} \mathrm{p}<0.05$, ${ }^{\dagger \dagger} \mathrm{p}<0.01$ vs. CKD. $\mathrm{c}$ Left, representative photomicrographs of immunohistochemistry assessing $\mathrm{ET}_{\mathrm{A}}$ protein expression in monkey kidney are presented. Right, densitometry data are presented as mean \pm standard error of the mean vehicle control. ${ }^{* *} \mathrm{p}<0.01$ vs. vehicle control. d NT-proBNP levels in monkeys treated with vehicle or bardoxolone methyl ( $30 \mathrm{mg} / \mathrm{kg} /$ day) for 28 days. Plasma samples were collected on day 28 at 1,2, 4, and $8 \mathrm{~h}$ after the dose (vehicle, $n=6$; BARD, $n=11$ ). Bars represent mean values \pm standard error of the mean with dots representing individual values. $* \mathrm{p}<0.05$ vs. vehicle control. 
change in either systolic or diastolic blood pressure, even at doses 4-fold higher than studied in BEACON. Intentto-treat analyses of phase $2 \mathrm{CKD}$ studies conducted with bardoxolone methyl showed no clear change in blood pressure across all patients enrolled, but these studies suggest that increases in both systolic and diastolic blood pressure were observed primarily in stage $4 \mathrm{CKD}$ patients and not in stage 3 CKD patients. Taken together, in parallel to sodium and water retention, these data suggest that the effects of bardoxolone methyl treatment on blood pressure may be associated with CKD severity.

Consistent with this phenotype for fluid retention, review of admission notes and narrative descriptions for heart failure hospitalizations in BEACON indicates that heart failure in bardoxolone methyl-treated patients was often preceded by documentation of other clinical symptoms or signs consistent with rapid fluid weight gain and was not associated with evidence of either acute renal decompensation or acutely reduced left ventricular contractility. The preserved ejection fraction observed in most patients at the time of hospitalization is consistent with clinical characteristics of heart failure caused by worsening diastolic dysfunction stemming from volume overload. Alternatively, a generalized increase in capillary permeability in the setting of acutely elevated blood pressure and/or a failure of an adequate pressure natriuresis response could explain clinically significant fluid retention. Notably, this collection of signs and symptoms differs from heart failure with reduced ejection fraction, which occurs because of left ventricular systolic impairment [23].

Collectively, the urinary electrolyte, BNP, and blood pressure data suggest that bardoxolone methyl treatment may differentially affect systemic hemodynamic status according to the clinical condition of subjects, having no clinically detectable effect in healthy volunteers or earlystage CKD patients, while apparently promoting fluid retention in patients with more advanced renal dysfunction and other recognized risk factors associated with heart failure at baseline.

Preclinical data suggest that bardoxolone methyl administration is associated with decreases in ET-1 secretion and $\mathrm{ET}_{\mathrm{A}}$ receptor expression. This modulation is distinct from endothelin receptor antagonists, which directly target the receptors that mediate the vascular effects of ET-1 in what would be considered a more classical drugreceptor interaction. Furthermore, unlike endothelin receptor antagonists, bardoxolone methyl does not have primary systemic hemodynamic effects that promote decreases in blood pressure. On the basis of these data, bar-

Bardoxolone Methyl Adverse Events Mechanisms doxolone methyl is currently being studied in patients with pulmonary arterial hypertension who do not have advanced renal dysfunction or other identified risk factors associated with heart failure at baseline (e.g. elevated BNP) [17].

Examination of renal-specific modulation of the endothelin pathway has been studied in animal models. Kidney-specific disruption of the endothelin pathway followed by salt challenge promotes fluid overload in knockout mouse models [24-26]. Moreover, the specific renal knockout of $\mathrm{ET}-1$ or $\mathrm{ET}_{\mathrm{A}}$ promotes fluid overload in animals with a clinical phenotype that is similar to endothelin receptor antagonist-mediated fluid overload in patients [25]. Notably, these animals experience acute increases in weight and blood pressure coupled with reduced urinary excretion of sodium and water. These effects are associated with acute activation of the epithelial sodium channel, which is expressed in the collecting ducts of the kidney, reabsorbs sodium, and promotes fluid retention [27]. Further, the endothelin pathway has been identified as a target of NF- $\mathrm{KB}$, with $\mathrm{ET}_{\mathrm{A}}$ expression decreased in renal artery organ cultures treated with direct NF- $\kappa \mathrm{B}$ inhibitors [28]. Thus, the inhibitory effects of bardoxolone methyl on the NF- $\kappa B$ pathway may at least possibly play a partial role on the effects on the endothelin pathway.

Plasma and urinary levels of ET- 1 indicate that urinary ET-1 levels are relatively unchanged in patients with eGFRs ranging from 100 to approximately $30 \mathrm{ml} / \mathrm{min} / 1.73$ $\mathrm{m}^{2}$; however, urinary ET-1 levels increase exponentially with decreasing eGFR in patients with stage 4 and 5 CKD [29]. These data suggest that renal ET-1 is primarily dysregulated in patients with advanced (stage 4 and 5) CKD.

Thus, the differential effects on fluid handling by bardoxolone methyl, if due to endothelin modulation, might be related to the disparate endogenous production of ET-1 in the kidney, which is meaningfully increased in stage 4 and 5 CKD patients [29]. Because endothelin signaling is dysregulated in the kidneys of patients with lower eGFR values, acute modulation of this pathway in a stage $4 \mathrm{CKD}$ patient population may have promoted acute sodium and water retention. As a result, the retention of fluid from bardoxolone methyl treatment may have translated to an increase in blood pressure and frank fluid overload in susceptible patients with baseline fluid retention, subclinical heart failure, and limited renal reserve. Importantly, bardoxolone methyl-mediated increases in eGFR may be due to glomerular effects, whereas effects on sodium and water regulation may be mediated by the tubules. As eGFR change was not correlated with heart 
failure, the effects of bardoxolone methyl on eGFR and sodium and water regulation appear to be anatomically and pharmacologically distinct. These mechanistic data, along with the clinical profile of bardoxolone methyl patients with heart failure, provide a hypothesis for defining the mechanism(s) of fluid retention in BEACON.

\section{Disclosure Statement}

G.L.B., P.A.M., D.P., and N.D.V. have received honoraria from Reata Pharmaceuticals for their involvement with the BEACON trial. D.G.W. is a consultant to, and has an equity interest in Reata. M.P.C., S.A.R., M.O.G., K.W.W., and C.J.M. are employed by and/ or have a financial interest in Reata. P.G.L. is employed by AbbVie.

\section{References}

1 Rojas-Rivera J, Ortiz A, Egido J: Antioxidants in kidney diseases: the impact of bardoxolone methyl. Int J Nephrol 2012;2012:1-11.

2 Yates MS, Tauchi M, Katsuoka F, Flanders KC, Liby KT, Honda T, et al: Pharmacodynamic characterization of chemopreventive triterpenoids as exceptionally potent inducers of Nrf2-regulated genes. Mol Cancer Ther 2007;6:154-162.

-3 Dinkova-Kostova AT, Liby KT, Stephenson KK, Holtzclaw WD, Gao X, Suh N, et al: Extremely potent triterpenoid inducers of the phase 2 response: correlations of protection against oxidant and inflammatory stress. Proc Natl Acad Sci USA 2005;102:4584-4589.

4 Lee D-F, Kuo H-P, Liu M, Chou C-K, Xia W, Du Y, et al: KEAP1 E3 ligase-mediated downregulation of NF-kappaB signaling by targeting IKKbeta. Mol Cell 2009;36:131-140.

5 Lisk C, McCord J, Bose S, Sullivan T, Loomis Z, Nozik-Grayck E, et al: Nrf2 activation: a potential strategy for the prevention of acute mountain sickness. Free Radic Biol Med 2013; 63:264-273.

6 Ahmad R, Raina D, Meyer C, Kharbanda S, Kufe D: Triterpenoid CDDO-Me blocks the NF-kappaB pathway by direct inhibition of IKKbeta on Cys-179. J Biol Chem 2006;281: 35764-35769.

7 Yore MM, Liby KT, Honda T, Gribble GW, Sporn MB: The synthetic triterpenoid 1-[2cyano-3, 12-dioxooleana-1, 9(11)-dien-28oyl]imidazole blocks nuclear factor-kappaB activation through direct inhibition of IkappaB kinase beta. Mol Cancer Ther 2006;5: 3232-3239.

8 Aminzadeh MA, Reisman SA, Vaziri ND, Khazaeli M, Yuan J, Meyer CJ: The synthetic triterpenoid RTA dh404 (CDDO-dhTFEA) restores Nrf2 activity and attenuates oxidative stress, inflammation, and fibrosis in rats with chronic kidney disease. Xenobiotica DOI: 10.3109/00498254.2013.852705.

-9 Aleksunes LM, Goedken MJ, Rockwell CE, Thomale J, Manautou JE, Klaassen CD: Transcriptional regulation of renal cytoprotective genes by Nrf2 and its potential use as a therapeutic target to mitigate cisplatin-induced nephrotoxicity. J Pharmacol Exp Ther 2010; 335:2-12.

$>10$ Wu QQ, Wang Y, Senitko M, Meyer C, Wigley WC, Ferguson DA, et al: Bardoxolone methyl (BARD) ameliorates ischemic AKI and increases expression of protective genes
Nrf2, PPAR $\gamma$, and HO-1. Am J Physiol Renal Physiol 2011;300:F1180-F1192.

11 Ding Y, Stidham RD, Bumeister R, Trevino I, Winters A, Sprouse M, et al: The synthetic triterpenoid, RTA 405, increases the glomerular filtration rate and reduces angiotensin II-induced contraction of glomerular mesangial cells. Kidney Int 2013;83:845-854.

12 Tanaka Y, Aleksunes LM, Goedken MJ, Chen C, Reisman SA, Manautou JE, et al: Coordinated induction of Nrf2 target genes protects against iron nitrilotriacetate (FeNTA)-induced nephrotoxicity. Toxicol Appl Pharmacol 2008;231:364-373.

13 Pergola PE, Raskin P, Toto RD, Meyer CJ, Huff JW, Grossman EB, et al: Bardoxolone methyl and kidney function in CKD with type 2 diabetes. N Engl J Med 2011;365:327-336.

14 De Zeeuw D, Akizawa T, Audhya P, Bakris GL, Chin M, Christ-Schmidt H, et al: Bardoxolone methyl in type 2 diabetes and stage 4 chronic kidney disease. N Engl J Med 2013; 369:2492-2503.

$\checkmark 15$ Pergola PE, Krauth M, Huff JW, Ferguson DA, Ruiz S, Meyer CJ, et al: Effect of bardoxolone methyl on kidney function in patients with T2D and stage 3b-4 CKD. Am J Nephrol 2011;33:469-476.

16 Reisman SA, Chertow GM, Hebbar S, Vaziri ND, Ward KW, Meyer CJ: Bardoxolone methyl decreases megalin and activates Nrf2 in the kidney. J Am Soc Nephrol DOI: 10.1681/ASN.2012050457.

17 Chin M, Wrolstad D, Bakris GL, Chertow GM, De Zeeuw D, Goldsberry A, et al: Risk factors for heart failure in patients with type 2 diabetes mellitus and stage 4 chronic kidney disease treated with bardoxolone methyl. J Cardiac Fail 2014, submitted.

18 De Zeeuw D, Akizawa T, Agarwal R, Audhya P, Bakris GL, Chin M, et al: Rationale and trial design of bardoxolone methyl evaluation in patients with chronic kidney disease and type 2 diabetes: The Occurrence of Renal Events (BEACON). Am J Nephrol 2013;37: 212-222.

19 Lambers Heerspink HJ, Chertow G, Akizawa T, Audhya P, Bakris G, Goldsberry A, et al: Baseline characteristics in the bardoxolone methyl evaluation in patients with chronic kidney disease and type 2 diabetes mellitus: the occurrence of renal events (BEACON) trial. Nephrol Dial Transplant 2013;28:28412850.
20 Kim HJ, Sato T, Rodríguez-Iturbe B, Vaziri ND: Role of intrarenal angiotensin system activation, oxidative stress, inflammation, and impaired nuclear factor-erythroid-2-related factor 2 activity in the progression of focal glomerulosclerosis. J Pharmacol Exp Ther 2011;337:583-590.

21 Skorecki KL, Winaver J, Abassi ZA: Extracellular fluid and edema formation; in Brenner BM (ed): Brenner and Rector's The Kidney, ed 8. Philadelphia, Saunders, 2008, pp 398446.

22 Mann JFE, Green D, Jamerson K, Ruilope LM, Kuranoff SJ, Littke T, et al: Avosentan for overt diabetic nephropathy. J Am Soc Nephrol 2010;21:527-535.

23 Vasan R, Larson M, Benjamin E, Evans JC, Reiss C, Levy D: Congestive heart failure in subjects with normal versus reduced left ventricular ejection fraction prevalence and mortality in a population-based cohort. J Am Coll Cardiol 1999;33:1948-1955.

24 Ahn D, Ge Y, Stricklett PK, Gill P, Taylor D, Hughes AK, et al: Collecting duct-specific knockout of endothelin-1 causes hypertension and sodium retention. J Clin Invest 2004; 114:504-511.

25 Evans RG, Madden AC, Oliver JJ, Lewis TV: Effects of ET(A) - and ET(B)-receptor antagonists on regional kidney blood flow, and responses to intravenous endothelin-1, in anaesthetized rabbits. J Hypertens 2001;19: 1789-1799.

26 Ge Y, Bagnall A, Stricklett PK, Strait K, Webb DJ, Kotelevtsev Y, et al: Collecting duct-specific knockout of the endothelin $\mathrm{B}$ receptor causes hypertension and sodium retention. Am J Physiol Renal Physiol 2006;291:F1274F1280.

27 Vachiéry J-L, Davenport A: The endothelin system in pulmonary and renal vasculopathy: les liaisons dangereuses. Eur Respir Rev 2009; 18:260-271.

28 Xie YH, Wang SW, Zhang Y, Evinsson L, Xu CB: Nuclear factor-kappaB-mediated endothelin receptor up-regulation increases renal artery contractility in rats. Basic Clin Pharmacol Toxicol 2013;113:401-410.

29 Dhaun N, Lilitkarntakul P, MacIntyre IM, Muilwijk E, Johnston NR, Kluth DC, et al: Urinary endothelin-1 in chronic kidney disease and as a marker of disease activity in lupus nephritis. Am J Physiol Renal Physiol 2009;296:F1477-F1483. 